tik im Vergleich zu anderen MINT-Fächern darstellt. Zu diesen Situationen gehören unter anderem Berufungskommissionen, die Drittmittelvergabe (z. B. in der DFG), sowie innerhalb einer Hochschule der Vergleich von Forschungsleistungen von Fachbereichen und für Gehaltsverhandlungen. In diesem Zusammenhang möchte ich darauf hinweisen, dass sich die DMV der „San Francisco Vereinbarung über die Forschungsbewertung" (DORA) (sfdora.org/read/de/) angeschlossen hat.
Abschließend möchte ich Sie ganz herzlich zur nächsten Jahrestagung der DMV vom 14.-17. September 2020 in Chemnitz einladen. Des weiteren möchte ich Sie auch auf die erste Gauß-Vorlesung des nächsten Jahres, die am 18. Juni 2020 in Greifswald stattfindet, aufmerksam machen.

Ich würde mich freuen, Sie auf diesen Veranstaltungen der DMV zu begrüßen und kennenzulernen.

Mit den besten Grüßen Ihr Friedrich Götze

\title{
Auslobung der Hermann Minkowski-Medaille 2020
}

Friedrich Götze hat es in seinem Grußwort für die Mitteilungen 27-2 (2019) bereits angekündigt: die DMV hat einen neuen Preis geschaffen, nämlich die Hermann Minkowski-Medaille. Die Einrichtung dieses Preises wurde auf der Mitgliederversammlung in Karlsruhe ausführlich diskutiert und einstimmig beschlossen. Der genaue Wortlaut des Beschlusses kann im Protokoll der Mitgliederversammlung, welches in diesem Heft auf Seite S. 162 zu finden ist, nachgelesen werden.

Bei diesem Preis geht es um eine Auszeichnung für Mathematikerinnen und Mathematiker, die sich zwar durch herausragende Arbeiten bereits internationale Anerkennung erworben haben, deren Karriere aber zu einem großen Teil noch vor ihnen liegt (Mid Career Prize). Konkret bedeutet dies, dass die Promotion nicht länger als zwölf Jahre zurückliegen soll, wobei Familienzeiten angemessen berücksichtigt werden. Die Zielgruppe, die wir hier adressieren wollen, ist damit vergleichbar mit der, welche auch durch den ERC Consolidator Grant angesprochen wird.

Bei der Benennung der Kandidatinnen und Kandidaten wollen wir neue Wege gehen. Mit der Ausschreibung der Minkowski-Medaille in diesem Heft bitten wir Sie um die Einreichung von Nominierungen (mit der üblichen Dokumentation) an die Geschäftsstelle der DMV. Gleichzeitig eröffnen wir die Möglichkeit der Eigenbewerbung. Der Termin für die Einreichung von Vorschlägen an die DMV Geschäftsstelle ist der 15. März 2020.

Mit diesem Preis will die DMV die Leistung der ausgewählten Preisträgerin oder des ausgewählten Preisträgers anerkennen und diese Leistungen auch über einen engeren wissenschaftlichen Kreis hinaus bekannt machen. Gleichzeitig soll dieser Preis eine Ermutigung für die weitere wissenschaftliche Tätigkeit sein. Daher bitten wir Sie, uns durch zahlreiche Nominierungen zu unterstützen.

Die DMV hat bereits eine Kommission eingesetzt, welche unmittelbar nach dem Eingang der Nominierungen ihre Arbeit aufnehmen wird. Die Hermann MinkowskiMedaille soll im zwei-jährigen Rhythmus, alternierend mit der Georg Cantor-Medaille, verliehen werden, und zwar erstmals auf der Jahrestagung 2020 in Chemnitz. Dort ist für die Preisträgerin oder den Preisträger bereits ein Vortragstermin reserviert.

An dieser Stelle möchte ich auch noch einmal auf den äußerst lesenswerten Artikel von Yuri Tschinkel über den Namensgeber dieses Preises „Geometrie der Zahlen - zum 150. Geburtstag von Hermann Minkowski“ (Mitteilungen $27^{-2}, 2019$ ) hinweisen. 